PAPER

\title{
Largeness and shape of sound images captured by sketch-drawing experiments: Effects of bandwidth and center frequency of broadband noise
}

\author{
Makoto Otani ${ }^{1, *}$, Kouhei Yamazaki ${ }^{2}$, Masahiro Toyoda ${ }^{3}$, \\ Masami Hashimoto ${ }^{4}$ and Mizue Kayama ${ }^{4}$ \\ ${ }^{1}$ Graduate School of Engineering, Kyoto University, \\ Kyoto daigaku-katsura, Nishikyo-ku, Kyoto, 615-8540 Japan \\ ${ }^{2}$ Graduate School of Science and Technology, Shinshu University, \\ 4-17-1 Wakasato, Nagano, 380-8553 Japan \\ ${ }^{3}$ Faculty of Environmental and Urban Engineering, Kansai University, \\ 3-3-35 Yamate-cho, Suita, 564-8680 Japan \\ ${ }^{4}$ Faculty of Engineering, Shinshu University, \\ 4-17-1 Wakasato, Nagano, 380-8553 Japan
}

(Received 28 September 2016, Accepted for publication 6 February 2017)

\begin{abstract}
When we listen to sounds radiated from a single sound source, the sound image is generally localized at a certain spatial position. In addition, similar to a visual image, the sound image has a certain largeness and shape. Previous works reported that a center frequency of the broadband noise affects the width of sound image; a bandwidth of broadband noise also affects the largeness of sound image. Although these works suggest that either the center frequency or frequency bandwidth has an impact individually on the largeness of sound image, an experiment employing simultaneous control of both parameters are required to explore how spectral characteristics of sound source signal affects the largeness of sound image. Furthermore, very little is known about the perception of the shape of sound image that would be essential for comprehensive understandings of spatial auditory perception. Therefore, in this work, a sketch-drawing experiment was conducted to capture the largeness and shape of sound image for a single sound source in an anechoic environment. The experimental results reveal that both the lower center frequency and the broader bandwidth of the broadband noise lead to the larger sound image. Moreover, the results show that inter- and intraindividual variations in the shape of sound image including circle, ellipse, and rectangular-like shape.
\end{abstract}

Keywords: Sound image, Largeness, Shape, Sketch drawing, Frequency bandwidth, Center frequency

PACS number: 43.66.Pn [doi:10.1250/ast.38.154]

\section{INTRODUCTION}

One can perceive a visual image of a visible object or environment through one's visual sensation. Similarly, one can perceive a sound image of an object that radiates sounds through one's auditory sensation. In general, the sound image is localized at a certain spatial position by using binaural and monaural localization cues such as interaural time differences (ITDs), interaural level differences (ILDs) and spectral cues [1]. Furthermore, the sound image would be perceived with a certain largeness and shape.

From the viewpoint of largeness of sound image, some perceptual measures such as auditory source width (ASW)

*e-mail: otani@archi.kyoto-u.ac.jp
[2] or spatial impression (SI) [3] were proposed. It is known that the ASW and SI vary with reflections from lateral direction to a listener. Then, lateral energy fraction $\left(L_{\mathrm{f}}\right)$ [4] and interaural cross correlation (IACC) [5-7] have been proposed as physical measures that are relevant to the ASW and SI. These discussions are limited to reflective or reverberant environments, because these researches were intended to provide knowledges for analysis and design of room acoustics.

As a physical factor, other than reflections, that would affect the largeness of sound image, Anazawa et al. suggested that the width of sound image for $1 / 3$ octaveband noise is affected by its center frequency; a lower center frequency yields a larger sound image [7], although their experiment did not explicitly assume a free field in which no reflections exist. Furthermore, the authors' 
previous work indicated that the largeness of sound image for a broadband noise of $1-\mathrm{kHz}$ center frequency vary with its frequency bandwidth; a broader bandwidth leads to a larger sound image in both the horizontal and vertical directions [8].

Although Anazawa et al.'s [7] and the authors' previous works [8] suggest that either the center frequency or frequency bandwidth has an impact individually on the largeness of sound image, more comprehensive experiments employing simultaneous control of both the center frequency and frequency bandwidth are required to further explore how spectral characteristics of sound source signal affect the largeness of sound image.

As described above, many researchers have been putting lots of efforts on clarification of relation between spatial positions of sound image and temporal and spectral characteristics of binaural signals, and some researchers have explored the largeness of sound image both in reflective and non-reflective environment. However, very little is known about the perceived shape of sound image, although it is quite intriguing to explore what "shape" the sound image has and how the physical characteristics of auditory input affects the shape. Potard et al. studied the apparent shape of sound sources, but they focused on a sound image generated by a set of multiple sound sources [9], not on a single sound source being of interest in the current work.

The experiments in the authors' previous work [8] measured the spatial extent of sound image only in the horizontal and vertical directions, thereby being not capable of capturing the shape of sound image. Therefore, in this work, for a single sound source in an anechoic environment, sketch-drawing experiments are employed to enable capturing the shape of sound image and its largeness.

Following sections are organized as below. Section 2 describes the method for subjective sketch-drawing experiment. Section 3 demonstrates the results and analysis of the experiment. Sections 4 and 5 respectively provide discussions and conclusions.

\section{METHOD}

A subjective experiment was performed to clarify how the center frequency and frequency bandwidth of broadband noise affect the perceived largeness and shape of sound image.

\subsection{Stimuli}

Stimuli were generated by convolving bandpass filters to a pink noise signal (sampling frequency: $44.1 \mathrm{kHz}$, 16-bit quantization). Nine bandpass filters (Butterworth, $3 \mathrm{~dB} /$ oct. attenuation) whose center frequencies are 500, 1,000 , and $2,000 \mathrm{~Hz}$ were prepared with bandwidths of 150 ,

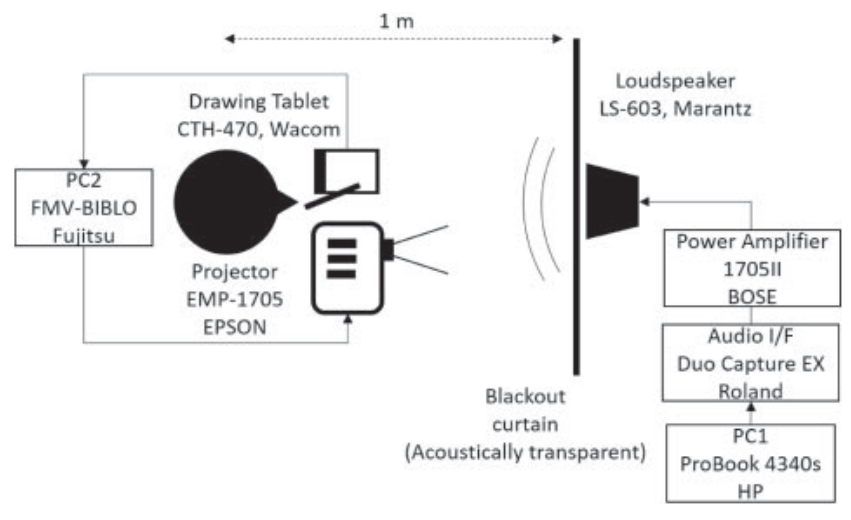

Fig. 1 Experimental system in an anechoic chamber.

300, and $600 \mathrm{~Hz}$ for each center frequency. The stimuli were presented through a loudspeaker. All the stimuli's amplitudes were adjusted so as to be approximately $60 \mathrm{~dB}$ of A-weighted sound pressure level when measured at a left ear of a head and torso simulator (SAMRAI, Koken).

\subsection{Procedure}

Participants were four males and one female, five in total, who reported no hearing difficulties. The experiment was conducted in an anechoic chamber. Figure 1 illustrates the experimental setups. The stimuli were output from a loudspeaker (LS603, Marantz) through a PC1 (ProBook 4340s, HP), the audio interface, and a power amplifier (1705II, BOSE).

The loudspeaker was located in front of the participant at 1-m distant. In order to remove effects of visual information, an acoustically transparent blackout curtain was located in front of the participant at 1-m distance.

The participants were equipped with a drawing tablet (CTH-470, Wacom) connected to PC2 (FMV-BIBLO NF/ G50, FUJITSU) that operates drawing software (Pixia 2.5). The canvas window of the drawing software was projected on the blackout curtain using a projector (EMP-1705, EPSON).

The participants were asked to draw outlines and other feature lines, if any, of sound image they perceived on the canvas projected on the blackout curtain by using the drawing tablet and an associated stylus pen while listening to the stimuli. Each stimulus was repeatedly presented until the participant finished drawing. The stimuli were presented in a randomized order. Five sessions were performed with intersession breaks where a single session consists of trials of 9 stimuli. Totally, 45 trials were conducted in a single day for each participant.

\section{RESULTS}

Figure 2 illustrates the examples of drawn sound images by the participant $\mathrm{C}$ for nine pairs of center 


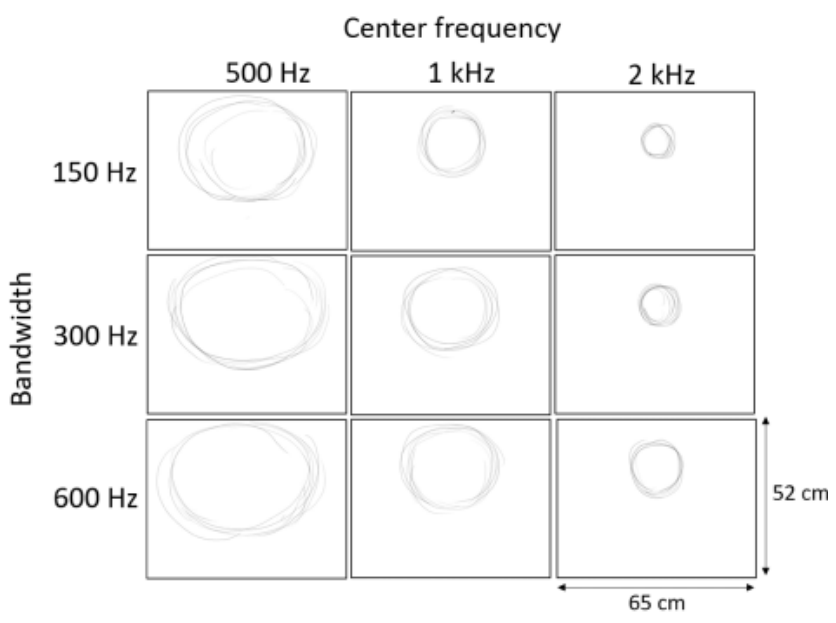

Fig. 2 Examples of drawn images by participant C.

frequency and bandwidth, which was chosen as a representative result for demonstrating variations due to frequency characteristics of source signals. Each panel corresponds to the canvas window of $65 \mathrm{~cm} \times 52 \mathrm{~cm}$ when projected to the blackout curtain. For each participant, from the drawn sound images, width and height in visual angle [deg.] and drawn area $\left[\mathrm{cm}^{2}\right]$ were calculated from the pixel area of the drawn image for each trial. Then, the resulting visual angles and drawn areas for five trials were averaged. Finally, averaged visual angles and drawn areas were obtained for nine conditions for each participant.

\subsection{Width and Height of Sound Image}

Figures 3(a) and 3(b) demonstrate the visual angles of the drawn sound image respectively in width and height, averaged among the participants. The abscissa and ordinate represent the frequency bandwidth $[\mathrm{Hz}]$ and the visual angle [deg.]. Error bars indicate standard errors. Both figures also present the results from previous experiment [8] which employed an adjustment method to measure sound image width and height. Note that the previous experiment was performed only for $1 \mathrm{kHz}$ of center frequency.

The two-way repeated-measures ANOVA (analysis of variance) revealed, for width, a significant main effect of the center frequency and, for height, significant main effects of the center frequency and the frequency bandwidth. No interactions between the center frequency and frequency bandwidth were found both for width and height. The multiple comparison test with the Bonferroni correction shows significant difference in the visual angles among bandwidths for each center frequency for 3 out of 9 cases respectively in width and height, indicated by the asterisks $\left(^{*}: p<0.05,{ }^{* *}: p<0.01\right)$.

Both in the current and previous experiments, the results demonstrate a common trend, namely, visual angles both in width and height are greater as the frequency

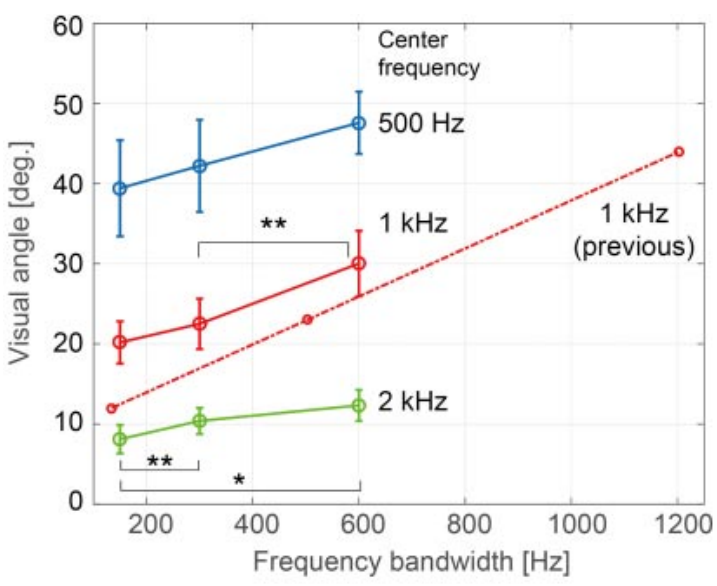

(a) Width vs frequency bandwidth.

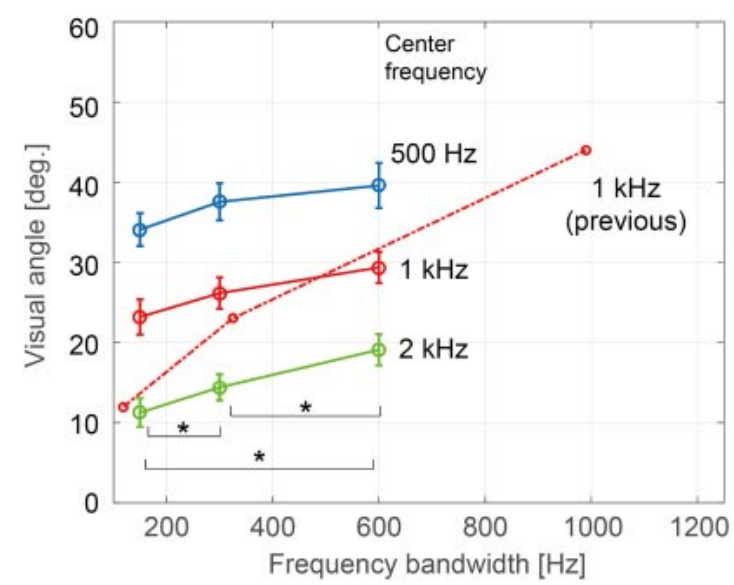

(b) Height vs frequency bandwidth.

Fig. 3 Visual angles in (a) width and (b) height vs. frequency bandwidth for each center frequency. Broken line indicates results from the previous experiment.

bandwidth increases. In addition, the current experiment shows that visual angles in both width and height are greater as the center frequency decreases. However, the results exhibits that visual angles in width are larger in the current experiment than in the previous one: visual angles in height in the current experiment are larger for narrower bandwidth and smaller for wider bandwidth than in the previous one. The reasons for these deviations between the current and previous results are yet to be revealed but they might be possibly caused by two different measurement methods, namely sketch drawing and adjustment methods.

For the current experiment, the results depict that, for $2-\mathrm{kHz}$ center frequency, the height is larger than the width for all the frequency bandwidths thereby indicating the drawn sound image is vertically long; for $1-\mathrm{kHz}$ center frequency, the height is larger than the width for 150 and $300 \mathrm{~Hz}$ of bandwidth and is almost same as the width for $600 \mathrm{~Hz}$ of bandwidth; for $500-\mathrm{Hz}$ center frequency, the height is smaller than the width for all the bandwidths indicating the drawn sound image is horizontally long. 


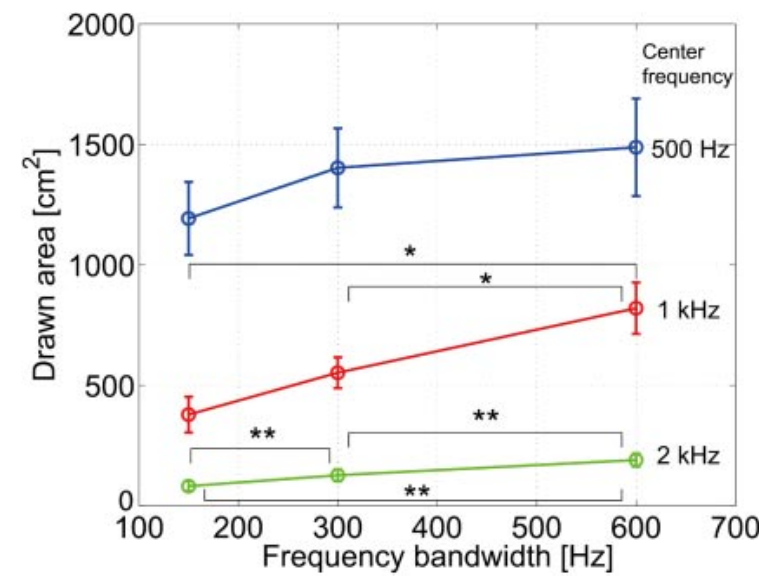

Fig. 4 Drawn area vs. frequency bandwidth for each center frequency.

\subsection{Largeness of Sound Image}

Figure 4 depicts the drawn area averaged among the participants. The abscissa and ordinate represent the frequency bandwidth $[\mathrm{Hz}]$ and the area of drawn sound image $\left[\mathrm{cm}^{2}\right]$ when projected to the blackout curtain. Error bars indicate standard errors.

The two-way repeated-measures ANOVA (analysis of variance) revealed significant main effects of the center frequency and frequency bandwidth. No interactions between the center frequency and frequency bandwidth were found. The multiple comparison test with the Bonferroni correction shows significant differences $(p<$ 0.05 ) of drawn area among all the three center frequencies for each bandwidth. Therefore, the results demonstrate that the drawn area is significantly greater as the center frequency decreases. The multiple comparison test with the Bonferroni correction shows that there are significant differences in the drawn area among bandwidths for each center frequency for 5 out of 9 cases indicated by the asterisks $\left(*: p<0.05,{ }^{* *}: p<0.01\right)$ in the figure. The results reveal that, for $2 \mathrm{kHz}$ and $1 \mathrm{kHz}$ of center frequency, the drawn area is greater as the frequency bandwidth increases; for $500 \mathrm{~Hz}$ of center frequency, the result shows a trend that the drawn area is greater as the bandwidth increases, although no significant differences are observed.

\subsection{Shape of Sound Image}

Figure 5 illustrates the examples of drawn images by five participants, which were chosen out of 5 trials for each participant so that they exemplify inter-individual variations in a comprehensible way. The figure depicts nine pairs of the center frequency and bandwidth for each participant.

\subsubsection{Inter-individual variation}

The participants $\mathrm{A}$ and $\mathrm{B}$ drew filled images while the participant $\mathrm{C}$ and $\mathrm{E}$ drew unfilled images having only outlines of the images. As the participants were just asked to draw outlines of the perceived sound images, it is likely that the participants $\mathrm{C}$ and $\mathrm{E}$ also perceived filled images but just left the images unfilled. Therefore, the differences between filled and unfilled images do not seem so meaningful. An interesting exception is the participant D who drew "cores" at the center of the unfilled circular images for all the trials. According to the results of participant D, it is likely that he/she perceived "dense" intensity in a small region at the center of "sparse" but broader circular sound images.

For most of the trials, the drawn sound images basically have elliptical shapes, including circle, and either vertically or horizontally long ellipse, depending on the participants, as demonstrated in Fig. 5. However, as shown in Fig. 6 illustrating drawn images from some of the trials of the participant $\mathrm{B}$, he/she drew rectangular-like shapes rather than elliptical one for some trials. Consequently, the results indicate that there are inter-individual variations in the perceived shape of sound image.

\subsubsection{Intra-individual variation in each condition}

Figure 7 illustrates the relative standard deviations (RSD) for each participant and each condition. The RSD is a standard deviation divided by an average value, expressing the extent to which a participant's response varies among 5 trials for a single condition. The ordinate denotes the RSD [\%]. The abscissa denotes the center frequency and bandwidth of the source signal along with a scale that indicates the largeness of drawn image area for each condition corresponding to the results demonstrated in Fig. 4. The figure demonstrates that the RSD value is generally larger with larger center frequency and smaller bandwidth, generally resulting in a smaller drawn image area. This indicates that the variance of drawn image largeness for same condition is relatively larger for smaller drawn images because the absolute variance is constant to some extent independent of drawn image largeness. It is yet to be revealed whether the variance of drawn image is attributable to ambiguity of perception of sound image largeness or that of drawing reproducibility in conversion from an auditory image to a visual image.

\subsubsection{Intra-individual variation among conditions}

Roughly speaking, as demonstrated in Fig. 5, the participants A, C, and D drew elliptical images that are slightly long in horizontal direction for lower center frequencies, while drawing circular images for higher center frequencies. In contrast, the participants B and E drew circular images and vertically long elliptical images depending on the stimulus for lower center frequencies, thereby demonstrating variations in the shape of drawn images. Especially, for the participant E, a higher center frequency results in a vertically longer ellipse. 


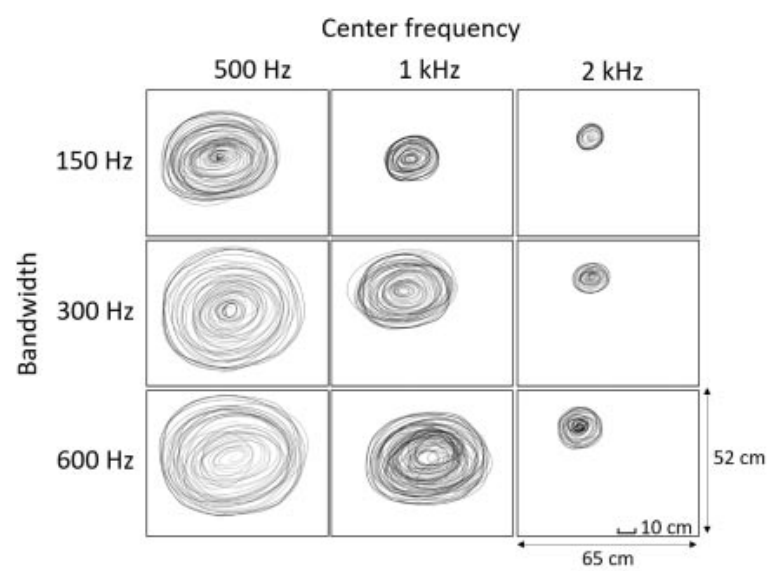

Participant A

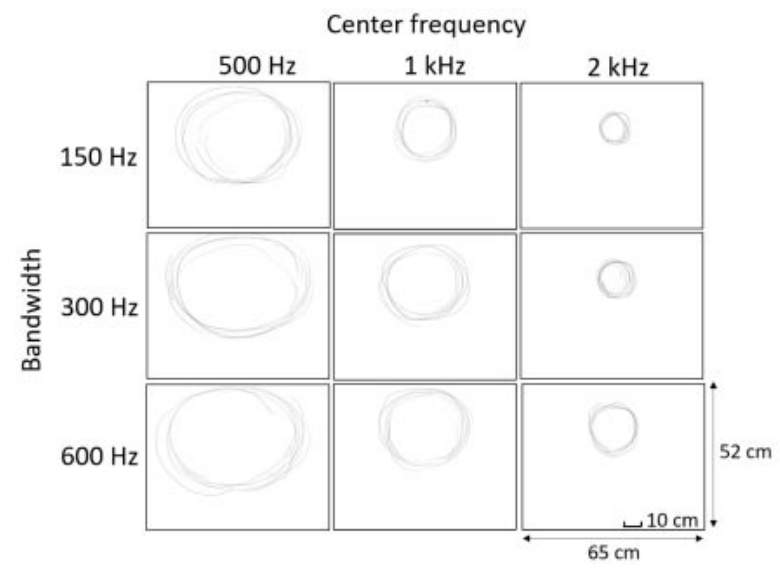

Participant C

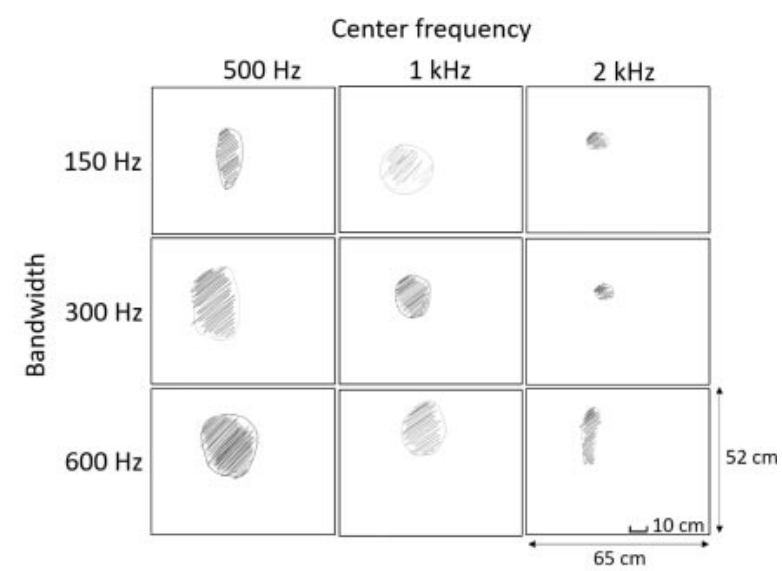

Participant B

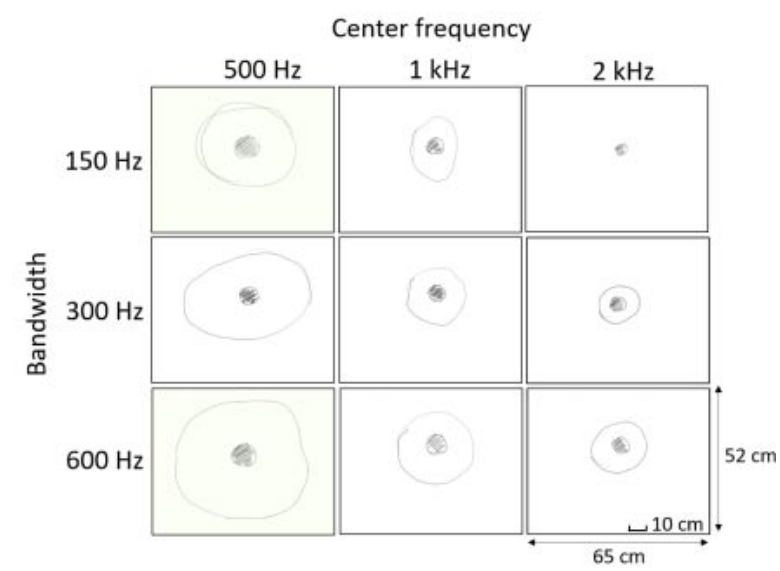

Participant D

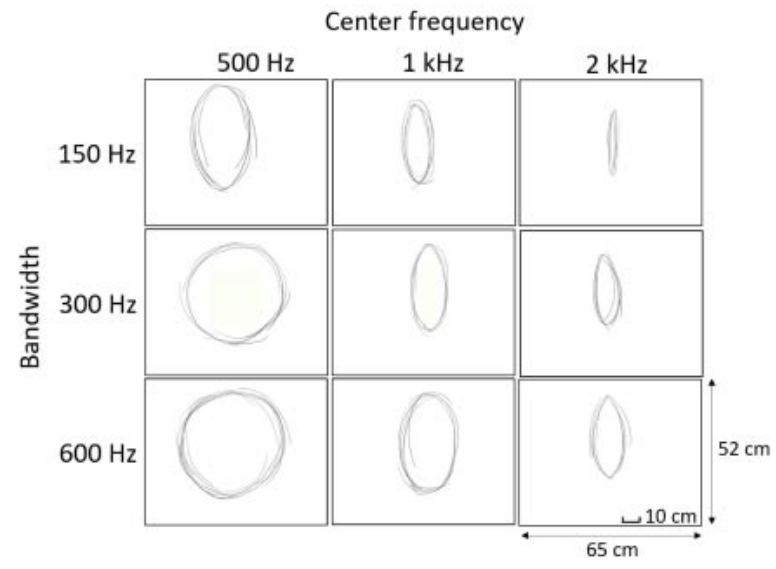

Participant E

Fig. 5 Examples of drawn images by each participant.

Consequently, the results demonstrate that there are intra-individual variations in the perceived shape of sound image depending on the center frequency and bandwidth.

\section{DISCUSSIONS}

If a sound image were always perceived as a circle, the largeness of sound image would be expressed by its width as a diameter of circle. However, a sound image can have an elliptical shape as observed in the current work, indicating that the largeness of the sound image cannot be expressed only by the width. Furthermore, for some cases, a sound image had a rectangular-like shape rather than an elliptical shape, as demonstrated in Fig. 6 from the participant B, as well as an elliptical shape with core, as in 


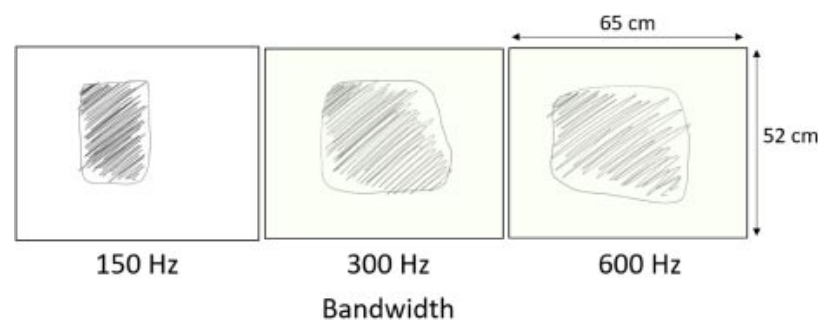

Fig. 6 Rectangular-like images drawn by participant B for $500-\mathrm{Hz}$ center frequency.

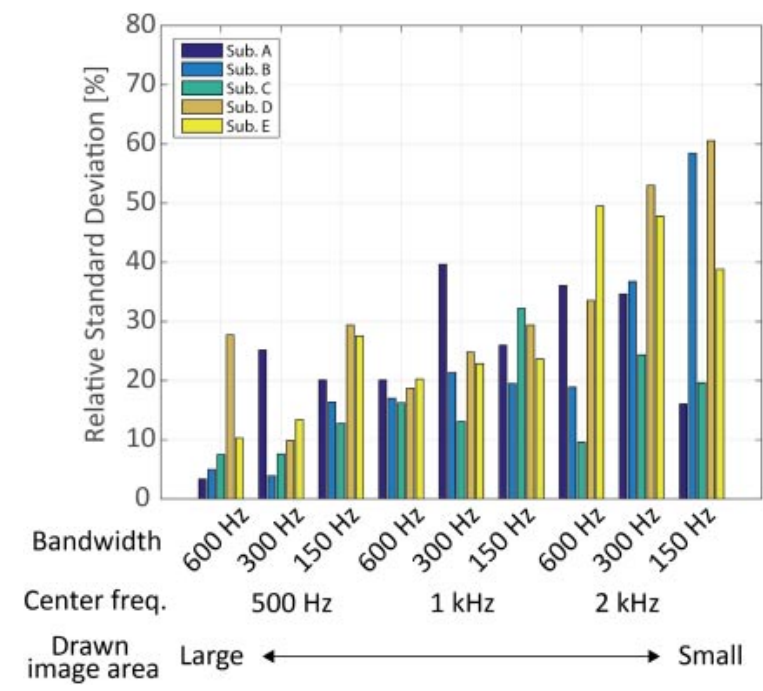

Fig. 7 Relative standard deviation [\%] for each participant and each condition.

Fig. 5. These results suggest that the sound images having non-simple shapes other than a circle or ellipse can be perceived; the sketch drawing experiment in the current work enabled the observation of sound image shape including such non-elliptic shapes while the measurement of width and height performed in the previous experiment [8] is insufficient for capturing such non-elliptic shape of perceived sound image.

Furthermore, the current results demonstrate that the shape of sound image varies among individuals, namely, not only the largeness but also the shape of sound image varies with the frequency characteristics of auditory input, i.e. the center frequency and bandwidth of the broadband noise in the current work. These findings imply that the perception of shape of sound images is a complicated phenomenon and requires further explorations. From the current results, it would be difficult to derive definitive explanations for intra- and inter-individual variations in the shape of sound image. However, if we could find any systematic variation from further subjective tests with larger number of participants, it may be possible to derive a reasonable and consecutive explanation for the intra- and inter-individual variations.

The ANOVA suggested no interactions between the center frequency and frequency bandwidth for width, height, and drawn area, which indicates that the center frequency and frequency bandwidth of the broadband noise individually affect the perception of width, height and largeness of sound image.

The current experiment employed the loudspeaker (LS603, Marantz) as a sound source. The loudspeaker has a 12-cm cone diameter, which may not be considered as a point source when it is located 1-m distant from a listener. However, the authors' previous result showed no significant difference in the perceived width and height of sound image between the loudspeaker presentation and the headphone presentation that presented an ideal point source through binaural synthesis [8], thereby indicating that, from the viewpoint of perception of sound image shape, a radiation pattern of the loudspeaker is not prominently different from an ideal point source. This indication raises a fundamental question whether the shape of wave front actually has an impact on the shape of sound image. If it does, further subjective experiments would be necessary in order to clarify how the shape of wave fronts does affect the perceived shape of sound image by employing various loudspeakers as well as an ideal planer source presented by binaural synthesis. If not, it would be intriguing to explore the reason why the participants perceived non-circular sound images including elliptical or rectangular-like ones. In addition, the future works would include investigations on sound image shape perceived by other stimulus signals such as pure tones, speech, or music signals.

\section{CONCLUSIONS}

A sketch-drawing experiment was performed to capture the effects of center frequency and bandwidth of the broadband noise on the largeness as well as the shape of sound images.

The experimental results revealed that both a lower center frequency and a broader bandwidth of broadband noise lead to a larger sound image; the center frequency and the frequency bandwidth can be individually considered in the perception of sound image largeness because no interactions are found between them.

Moreover, the results show that inter- and intraindividual variations in the shape of sound image such as a circle, vertically and horizontally long ellipses and rectangular-like shapes.

\section{ACKNOWLEDMENT}

This work was partially supported by a Grant-in-Aid for Scientific Research (B) (26280078 and 16H02857), MEXT, Japan. 


\section{REFERENCES}

[1] J. Blauert, Spatial Hearing: The Psychophysics of Human Sound Localization (MIT Press, Cambridge, MA, 1997).

[2] W. V. Keet, "The influence of early lateral reflections on the spatial impression," Proc. 6th Int. Congr. Acoust., E-2-4, E-53E-56 (1968).

[3] M. Barron, "The subjective effects of first reflections in concert halls-The need for lateral reflections," J. Sound Vib., 15, 475494 (1971).

[4] M. Barron and A. H. Marshall, "Spatial impression due to the early reflections in concert halls: The deviation of a physical measure," J. Sound Vib., 77, 221-232 (1981).

[5] M. Morimoto, K. Iida and Y. Furue, "Relation between auditory source width in various sound fields and degree of interaural cross-correlation," Appl. Acoust., 38, 291-301 (1993).

[6] K. Kurozumi and K. Ohgushi, "Relation between crosscorrelation coefficient of two channel acoustic signals and sound image quality," J. Acoust. Soc. Jpn. (J), 39, 253-260 (1983) (in Japanese).

[7] T. Anazawa, H. Yanagawa and T. Itow, "On correlation coefficients of both ears and "Feeling of Wideness"," Tech. Rep. Inst. Electr. Commun. Eng. Jpn., EA70-13 (1970).

[8] M. Otani, K. Yamazaki, M. Toyoda, M. Hashimoto and M. Kayama, "Relation between frequency bandwidth of broadband noise and largeness of sound image," Acoust. Sci. \& Tech., 38, 35-37 (2017).

[9] G. Potard and I. Burnett, "A study on sound source apparent shape and wideness," Proc. Int. Conf. Auditory Display, Boston, pp. 25-28 (2003). 